\title{
NEONATAL SURGERY: DEMAND AND SURVIVAL BOTH ARE INCREASING: EIGHT YEARS EXPERIENCE IN DHAKA MEDICAL COLLEGE HOSPITAL, BANGLADESH
}

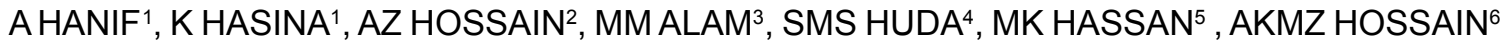

\begin{abstract}
:
Purpose: In order to achieve the Millennium Development Goal (MDG) 4 target of a two-thirds reduction in under-five mortality from 1990 to 2015, major reductions are going to be required in neonatal mortality. Congenital anomalies have become the fourth cause of neonatal deaths and most of these are curable. Dhaka Medical College Hospital is the largest public hospital of the country and serving the poor and lower middle class people where surgery and medical facilities are mostly free of cost. This study was done to see the types of neonatal surgical patients admitted in this hospital and their management out come with limited facilities and find out the ways to improve the scenario to contribute in achieving the MDG.
\end{abstract}

Materials \& Methods: This was a retrospective study done over a period of 8 years from July 2001 to June 2009 and carried out in the Department of Pediatric Surgery, Dhaka Medical College Hospital, Dhaka. A total of 650 neonates were admitted during this period and it was the $17.99 \%$ of total number of $\mathbf{3 6 1 2}$ pediatric surgical admission upto12 years of age. Data was collected from hospital records and analyzed retrospectively.

Results: Out of these 650 neonates $533(80 \%)$ were admitted for Neonatal Intestinal Obstruction (NIO) and

1. Dr. Abdul Hanif, Dr. Kaniz Hasina, Assistant Professor, Department of Pediatric Surgery, Dhaka Medical College \& Hospital, Dhaka 1000, Bangladesh

2. Dr. Ahmed Zahid Hossain, Associate Consultant, Department of Pediatric Surgery, Square Hospitals Ltd.

3. Dr. S M Shamsul Huda, Assistant Registrar, Department of Pediatric Surgery, Dhaka Medical College \& Hospital, Dhaka 1000, Bangladesh

4. Dr. M Kamrul Hassan, Assistant Professor, Department of Pediatric Surgery, Rajshahi Medical College \& Hospital, Rajshahi

5. Dr. Md. Mahbub-UI-Alam, Professor, Department of Pediatric Surgery, Dhaka Medical College \& Hospital, Dhaka 1000, Bangladesh

6. Dr. AKM Zahid Hossain, Assistant Professor, Department of Pediatric Surgery, Banbanandhu Sheikh Mujib Medical University, Dhaka

Correspondence to: Dr. Abdul Hanif (Tablu), Assistant Professor, Department of Pediatric Surgery, Dhaka Medical College \& Hospital, Dhaka 1000, Bangladesh, E-mail: ahaniftablu@hotmail.com omphalocele were $59(9.07 \%)$, ectopia vesicae are 14 $(2.15 \%)$, posterior urethral valves (PUV) were $19(2.92 \%$ ), gastroschisis were $7(1.07 \%)$, congenital diaphragmatic hernia (CDH) were $16(2.46 \%)$,Tracheo-oesophageal fistula $1(0.15 \%)$, and Conjoint two $1(0.15 \%)$. . The most common cause of NIO was anorectal malformation (ARM) and $194(66.43 \%)$ patients have high variety and $98(33.56 \%)$ patients have low variety ARM. Next was Hirschsprung disease and $137(21.07 \%)$ neonates presented with this. Thirty-Five $(5.38 \%)$ patients presented with septicemia and $40(6.15 \%)$ presented with intestinal atresia, 20 $(3.07 \%)$ neonates had meconium ileus and $9(1.38 \%)$ patients presented with volvulus neonatorum. Five hundred and Fifty-nine patients $(86.00 \%)$ were managed surgically. Out of 650 patients, 69 died, so mortality was $10.61 \%$ and before surgery $2.76 \%$ and after surgery was $7.84 \%$.

Conclusion: Pediatric surgeons by their skill and teamwork greatly improved the neonatal surgical service and contributing significantly in reducing infant mortality rate and to achieve MDG 4 . But to improve further, neonatal intensive care unit (NICU) and other support systems are essential as well as support from UNICEF and World Health Organization (WHO) to include pediatric surgery and surgeons in their activities especially in developing countries. Due to socio-political and economic reasons of the developing countries the roll of pediatric surgeons are multidimensional.

\section{Introduction:}

Over one third of the global 10.8 million deaths of children under age 5 in the year 2000 occurred in the neonatal period $^{1}$. Declines in neonatal mortality over the last three decades have been slower than declines in post neonatal or early child ( 1 to 5 years) mortality. In order to achieve the Millennium Development Goal target of a two-thirds reduction in under-five mortality from 1990 to $2015^{2}$, major reductions are going to be required in neonatal mortality ${ }^{3}$. Congenital anomalies have become the 4th cause of neonatal death and most of these are curable ${ }^{4}$. Population of Bangladesh is approximately 
142 million and about 12.3 million people lives in Dhaka. Bangladesh has a stable, growing economy, but living standards have yet to improve for the poorest and most vulnerable segments of the population. About 36 percent of the population lives on less than $\$ 1$ a day $^{5}$. Dhaka Medical College Hospital is the largest public hospital of the country and serving these poor and lower middle class people where surgery and medical facilities are mostly free of cost. This study has been done to see the types of neonatal surgical patients admitted in this hospital and their management out come with limited facilities and find out the ways to improve the scenario to contribute in achieving MDG4.

Neonatal intestinal obstruction (NIO) is the most common form of neonatal emergencies in neonatal surgical patients. It is caused by a group of congenital anomalies and some other acquired conditions ${ }^{6}$. Neonatal surgeries require surgical management by pediatric surgeons in medical centers with facilities for pediatric anesthesia, radiology and the specialized nursing care for successful survival ${ }^{7,8}$.

The principal feature of neonatal intestinal obstruction is bile-stained vomiting. Early vomiting, in the first $24 \mathrm{~h}$ of life, indicates a high obstruction (duodenal or jejunal) while the later onset of vomiting indicates a lower obstruction (ileal or colonic) ${ }^{8-9}$. Failure to pass me conium ( $48 \%$ of normal neonates pass me conium in the first 24 $\mathrm{h}$ of life) is symptomatic of lower intestinal obstruction ${ }^{10-}$ 12. The degree of abdominal distension correlates roughly with the height of the intestinal obstruction. In duodenal and high jejunal obstruction the distension is restricted to the upper abdomen, whereas in ileal and colonic obstruction there may be massive, unrestricted distension ${ }^{13-15}$. Waves of peristaltic movement may be visible during an abdominal examination of a neonate with intestinal obstruction.

Maternal polyhydramnios, dilated fluid filled loops of gut at maternal ultrasonography and family history of cystic fibrosis or Hirschsprung's disease are the antenatal diagnostic tool for NIO. It is an urgent condition that requires immediate involvement of a team of pediatric surgeon and neonatologist for peri-operative management. Fluid loss, electrolyte imbalance, metabolic acidosis, hypoglycemia, hypothermia, respiratory distress and sepsis lead to high morbidity and mortality due to intestinal obstruction ${ }^{16}$.

The Others Congenital anomalies we have treated were Omphalocele, Posterior urethral valve, Congenital Diaphragmatic Hernia, Ectopia Vesicae, and Gastroschisis.

\section{Materials and Methods:}

This was a retrospective study done over a period of 8 years from July 2001 to June 2009 and carried out in the Department of Pediatric Surgery, Dhaka Medical College and Hospital, Dhaka. A total of 650 neonates were admitted during this period and it was the $17.99 \%$ of total pediatrics surgical admission. Out of these 650 neonates, 533 were admitted for neonatal intestinal obstruction (NIO). Among them, 366 were male and 283 were female. Parameters of clinical presentations at the time of admission, measures of resuscitation and peri-operative measures were taken and recorded. Diagnoses of the patients were based on detailed history, complete physical examination and laboratory and radiological findings. Surgical interventions were undertaken as soon as possible after appropriate diagnosis and proper resuscitation. Local anesthetic $(0.5-1.0 \mathrm{ml} / \mathrm{kg}$, both lignocain and bupivacain) was given in incision line as pre-emptive analgesia in all cases.

\section{Results:}

Total number of neonate admitted during the study period was 650 neonates having Surgical Problems. The age ranges of the neonates ranged from 2 days to 28 days with the mean age of $8.1 \pm 4$ days. Weight ranged from 1.5 to $3.5 \mathrm{~kg}$. Majority of the patients came from poor socioeconomic status. Male: female ratio was 1.3: 1.

The major clinical presentations of the patients included abdominal distension $(78.15 \%)$, bilious vomiting $(60.30 \%$ and failure to pass me conium $(75.07 \%)$ (Table-I). Some patients exhibited dehydration $(23.38 \%)$ and fever $(20.92 \%)$, Abdominal wall defect (11.53\%), Dribbling of urine $(8 \%)$ and Respiratory distress (2.46\%).

Table-I

Clinical presentations of neonatal surgical patients)

$$
(n=650)
$$

\begin{tabular}{lcc}
\hline Presentation Frequency, & No. & $(\%)$ \\
\hline Failure to pass me conium & 488 & $(75.07)$ \\
Abdominal distension & 508 & $(78.15)$ \\
Bilious vomiting & 392 & $(60.30)$ \\
Dehydration & 152 & $(23.38)$ \\
Fever & 136 & $(20.92)$ \\
Dribbling of Urine & 52 & $(8.00)$ \\
Abdominal Wall Defect. & 75 & $(11.53)$ \\
Respiratory Distress. & 16 & $(2.46)$ \\
\hline
\end{tabular}

The most common variety of neonatal surgical patients were anorectal malformation (ARM) 292. and among this 194 (66.43\%) patients had high variety and $98(33.56 \%)$ patients had low variety ARM (Table II). This was followed next by Hirschsprung disease that was presented by $137(21.71 \%)$ of the cases. 
Omphalocele $59(9.07 \%)$, Intestinal atresia 40 $(6.15 \%)$, Septicemia $35(5.38 \%)$, Congenital Diaphragmatic hernia 16 (2.46\%), Meconium ileus 20 (3.07\%), Posterior Urethral Valve 19 (2.92\%)

Table-II

Variety of Neonatal Surgical Patients $(n=650)$

\begin{tabular}{lcc}
\hline Cause Frequency, & No & $(\%)$ \\
\hline Anorectal malformation (ARM) & 292 & $(44.92)$ \\
Hirschsprung's disease & 137 & $(21.07)$ \\
Intestinal atresia & 40 & $(6.15)$ \\
Septicemia & 35 & $(5.38)$ \\
Omphalocele & 59 & $(9.07)$ \\
Ectopia Vesicae & 14 & $(2.15)$ \\
Congenital Diaphragmatic Hernia & 16 & $(2.46)$ \\
Posterior Urethral Valve & 19 & $(2.92)$ \\
Meconium ileus & 20 & $(3.07)$ \\
Gastroschisis & 7 & $(1.07)$ \\
Volvulous neonatoram. & 9 & $(1.38)$ \\
Tracheo-oesophageal fistula & 1 & $(0.15)$ \\
Conjoint twin. & 1 & $(0.15)$ \\
\hline
\end{tabular}

Thirty-Five. (5.38\%) patients presented with septicaemia and $40(6.15 \%)$ presented with intestinal atresia (Fig.IV). Another $20(3.07 \%)$ patients had meconium ileus and $9(1.38 \%)$ patients presented with volvulus neonatorum. Five hundred and Fifty-nine patients (86.0\%) were managed surgically (Table-III). Out of 650 patients, 69 died (Table-IV). The mortality rate was $10.61 \%$ including $2.76 \%$ before surgery and $7.84 \%$ after surgery.

Table-III

Treatment modalities of the patients $(n=650)$

\begin{tabular}{lcc}
\hline Treatment modalities Frequency, & No. & $(\%)$ \\
\hline Pelvic colostomy & 194 & $(29.84)$ \\
Laparotomy, labelling biopsy & 76 & $(11.69)$ \\
and colostomy & & \\
Anoplasty & 98 & $(15.07)$ \\
Rectal biopsy and colostomy & 51 & $(7.84)$ \\
Primary Repair & 32 & $(4.92)$ \\
Cutaneous Vesicostomy & 14 & $(2.15)$ \\
lleostomy & 11 & $(1.69)$ \\
Laparotomy and Repair & 14 & $(2.15)$ \\
Laparotomy, resection and primary & 69 & $(10.61)$ \\
anastomosis & & \\
Conservative & 91 & $(14)$ \\
\hline
\end{tabular}

Table-IV

Morbidity and mortality of the patients $(n=650)$

\begin{tabular}{lrr}
\hline Morbidity and mortality Frequency, & No. & $(\%)$ \\
\hline After surgical intervention & & \\
$\quad$ Wound infection & 62 & $(9.53 \%)$. \\
$\quad$ Death. & 51 & $(7.84 \%)$ \\
After conservative treatment & & \\
$\quad$ Death. & 18 & $(2.76 \%)$ \\
$\quad$ Total death & 69 & $(10.61 \%)$. \\
\hline
\end{tabular}

Table-V

During Last Two Years (July2007 to June 2009):

\begin{tabular}{lc}
\hline Total Admission up to 12 years. & 1071. \\
Total Neonatal Admission & 220 \\
Percentage of Neonatal Admission & $20.54 \%$ \\
Total Death & $18(8.18 \%)$ \\
Before Surgery & $7(3.18 \%)$ \\
After Surgery & $11(5 \%)$ \\
\hline
\end{tabular}

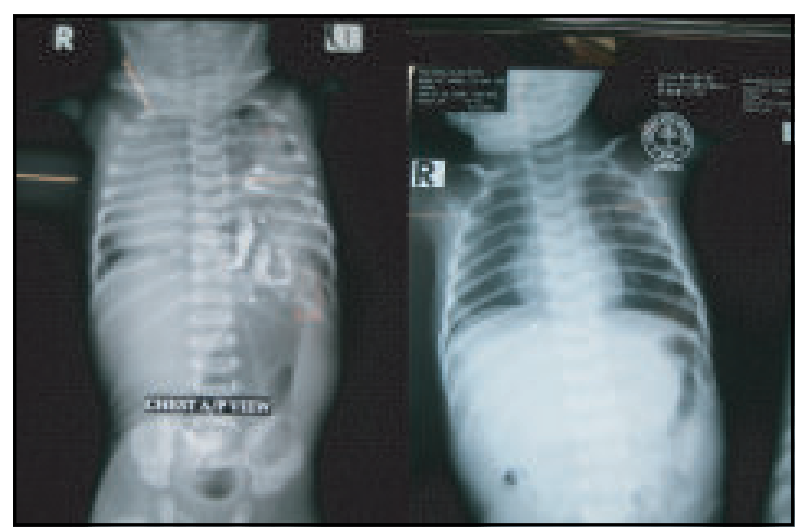

Fig.-1: Diaphragmatic Hernia (Preoperative and Post operative Picture)

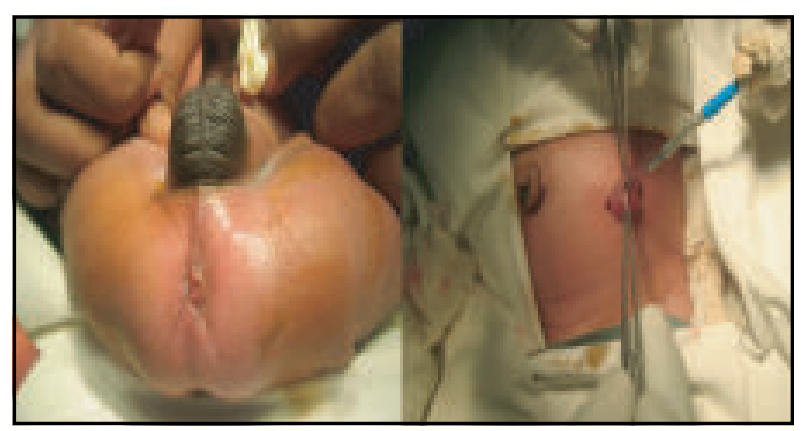

Fig.-2: ARM (Preoperative and Peroperative procedure) 


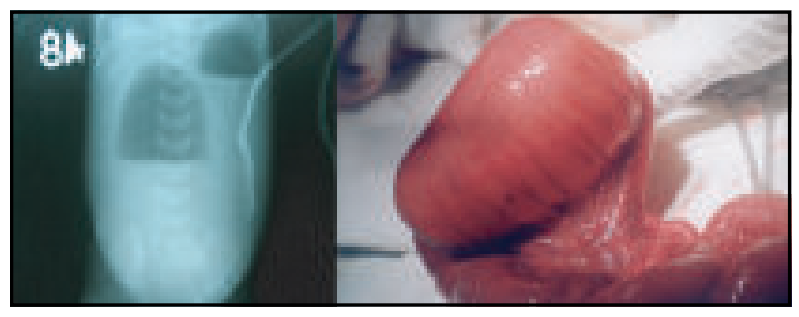

Fig.-3: Duodenal atrisia (Double bubble appearance \& Peroperative view)

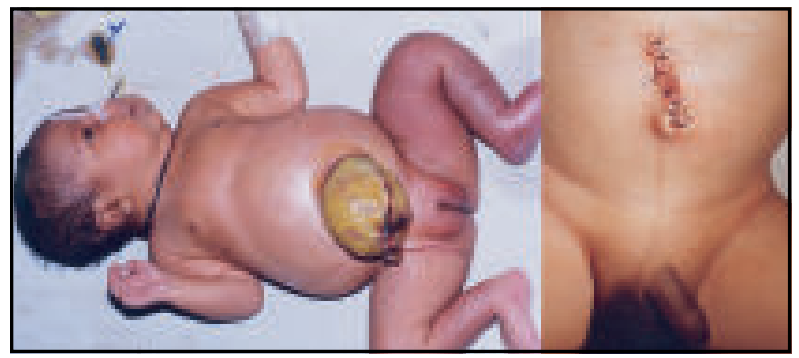

Fig.-4: Omphalocele (Preoperative and postoperative view)

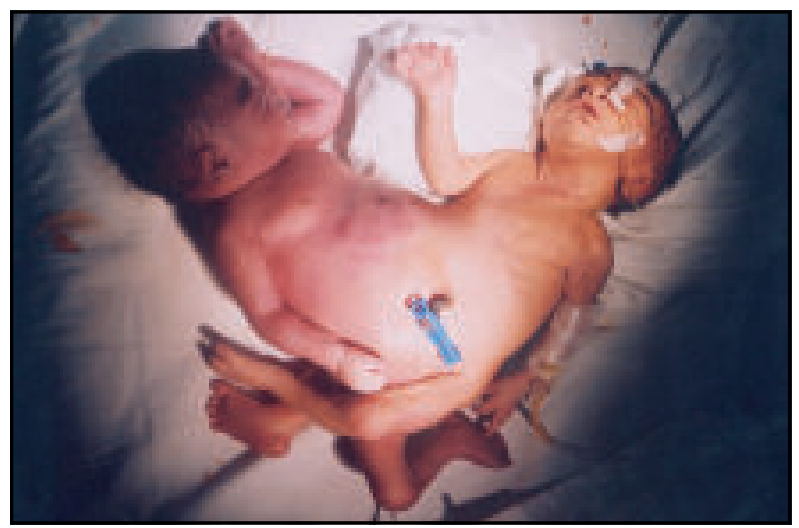

Fig.-5: Conjoined Twin

\section{Discussion:}

Pregnancy and childbirth are generally times of joy for parents and families. In Bangladesh parents distribute sweets after a healthy child is borne. But like many countries and communities, they are also periods of great risk to the health and survival of women and newborns. Every day, around 1500 women die from complications related to pregnancy and childbirth. The divide between the industrialized countries and developing regions, particularly the least developed countries, is perhaps greater on maternal mortality then on any issue. The average lifetime risk of a woman in a least developed country dying from complications related to pregnancy or childbirth is more than 300 times greater than for a woman living in an industrialized country. Likewise earliest days of life are the most vulnerable for a child. Almost 40 percent of under-five deaths - numbering 3.7 million in 2004, the latest year for which firm estimates are available - take place during the first 28 days, the neonatal period. Three quarters of these neonatal deaths take place during the first 7 days. Health divide in neonatal mortality is also marked; a child born in a least developed country is almost 14 times more likely to die during the first 28 days of life than a child born in an industrialized country. And these burden of death are heavy for Africa and Asia, accounts for 95 per cent maternal and 90 per cent newborn deaths ${ }^{17}$.

In neonates mortality is decreasing in medical causes like severe infection (including pneumonia, sepsis, diarrhoea), asphyxia and preterm births, mortality is now increasing in surgical causes including congenital anomalies. Most of these congenital anomalies are surgically curable and more than 85 to 90 percent of these neonates are surviving with limited facilities which two decades back more than 50 per cent died when there was no pediatric surgical unit in most of the medical college hospitals of Bangladesh and general surgeons did the surgeries ${ }^{18}$. After establishing pediatric surgical unit in Dhaka Medical College Hospital in 1993 pediatric surgeons are contributing gradually in the reduction of neonatal mortalities and as a whole child mortalities. In this study different types of neonatal surgical problems, their management and out come have been shown.

Neonatal intestinal obstruction (NIO) was the most common form of neonatal emergencies. It was caused by a group of congenital anomalies and some other acquired conditions. NIO presented with a triad of bile stained vomiting, abdominal distension and failed to pass meconium6. Maternal polyhydramnios, dilated fluid filled loops of gut at maternal ultrasonography, postnatal bilious vomiting with or without abdominal distension, plain X-ray of abdomen shows distension of the gut with air fluid level requires immediate attention and evaluation involving of a team of pediatric surgeons and neonatologists for peri-operative management ${ }^{11}$.

A neonatal abdominal problem may come to light in a newborn baby in a variety of way. Maternal polyhydramnious, increased volume of nasogastric aspirate, passes of per-rectal bleeding, abdominal distension, apnoeic episodes and failure to pass me 
conium $^{12}$. Etiology of neonatal intestinal obstruction is varied, mostly congenital and some are acquired. Congenital varieties always treated by surgical treatment and acquired ones mostly treated conservatively. Sometimes like neonatal necrotizing enterocolities should be treated by surgical intervention.

Omphalocele: Total 59 patients were admitted during the study period. 12 patients were treated surgically. Out of them 5 patients were died preoperatively and 4 patients were died postoperatively due to infection, membrane rupture \& other congenital anomalies. 47 patients were managed conservatively with $0.5 \%$ mercurochrome, alcohol, povidone iodine. After escherification of omphalocele membrane it converted into ventral hernia, which corrected later on. The result of conservative management (in absence of complications demanding immediate surgery) of omphalocele major followed by delayed closure of the ventral hernia is encouraging in situations where facilities for neonatal intensive care, parental nutriotional support and readymade artificial covering materials (silo) for surgical closure are not available. ${ }^{19}$

Posterior Urethral Valve: Total 19 Patients were admitted. Due to absence of neonatal cystoscope cutaneous vesicostomy were done on 14 patients and catheterizations were done on 5 patients for temporary relief of symptoms. Definitive treatment was done later on. Recently pediatric surgery unit has collected a $8 \mathrm{~F}$ cystoscope and pediatric urological services are improved \& increased.

Congenital Diaphragmatic Hernia : Total 16 patients were admitted.. Out of them 3 patients died preoperatively during resuscitation. Thirteen neonates were treated surgically and 2 patients were died postoperatively due to unsuccessful recovery from anesthesia \& lack of NICU support. Outcome of other patients were good. Survival rate was $68.75 \%$. The largest multicenter report on $\mathrm{CDH}$ mortality to date is that of the CDH Study Group, which has reported an overall survival of $68 \% 20$. In our country the CDH patients with severe pulmonary hypoplasia do not get chance to attend the hospital. They may die after birth or before attending any doctor. Even sometimes it is not diagnosed at all. The $\mathrm{CDH}$ patients who are adopted their respiratory physiology and do not developed the consequence of $\mathrm{CDH}$ come to our hospital only with respiratory distress and some times with features of intestinal obstruction. Possibly for this reason the survival rate is higher in this study. ${ }^{21}$

Ectopia Vesicae: Total 14 patients were treated during study period. All of them were treated surgically by primary closure of anterior urinary bladder wall \& anterior abdominal wall. 5 patients were developed postoperative wound dehiscence. Outcome of other neonates were uneventful.

Gastroschisis: A number of patients were visited as emergency basis \& 7 patients were admitted \& other were referred on demand of parents. Out of them 3 were died preoperatively during resuscitation \& 4 patients were died postoperatively due to inadequate reversal from anesthesia \& lack of NICU support.

Conjoint Twin \& Esophageal Atresia: One surgery for esophageal atresia with distal tracheo-oesophageal fistula was performed, reversal was good but the unfortunate baby died on second postoperative day due to lack of NICU support. Department's surgical team on emergency basis also separated a conjoint twin from its dead sibling but the baby died two hours after operation in the way for NICU support.

Among 650 patients, 559 (86.00\%) were treated by surgical intervention. Out of 559 cases, 535 had uneventful recovery and 24 patients had delayed recovery, 62 patients developed wound infection and 69 patients died. Among these 69 deaths, 8 patients did not recovere from anesthesia. Ninety one (14.0\%) patients were treated conservatively, out of which 73 were improved and 18 died. Presentation, diagnosis and treatment modalities of this study are more or less consistent with the other studies ${ }^{22,23}$. Fluid loss and electrolyte imbalance, metabolic acidosis, hypoglycemia, hypothermia results from inadequate warming during examination and/or negligence, respiratory distress and septicemia are the leading factors for high morbidity and mortality of intestinal obstruction $^{24}$.

In case of neonate diaphragm is the only respiratory muscle for respiration, which is abdomino-thorasic. After laparotomy wound closure abdominal movement becomes restricted during reversal and postoperative period due to pain in incision line. In this study, all cases were given pre-emptive analgesia with 0.5-1$\mathrm{ml} / \mathrm{kg}$ local anesthetic (both lignocain and bupivacain) in incision line before the incision was made. So for this simple but good pain management technique recovery from anesthesia was good as well as postoperative period was uneventful without any 
ventilatory support ${ }^{25}$-which this hospital could not provide due to absence of NICU. Thus this technique helps in reducing mortality.

During last two years (July/07 to June/09) neonatal surgical services and surgery increased than the previous years. Mortality also reduced. Another study of 6 years showed that average admission per year was 72 and mortality was $14.8 \%{ }^{26}$. In July 2007 to June 2009 average admission per year is 110 and mortality is $8.18 \%$ (Table- $V$ ). The cause of morbidity and mortality were due to pre-existing conditions including co-morbidity, septicemia and also some postoperative complications. In spite of our limited resources and absence of NICU support this series showed a good outcome.

\section{Conclusion:}

In developing countries the role of pediatric surgeons are multidimensional due to socio-political and economic reasons. Pediatric surgeons by their skill and teamwork greatly improved the neonatal surgical services and contributing significantly in reducing infant as well as neonatal mortality rate and thus help in achieving MDG4. But to improve further NICU, other support systems are very essential. It is advisable that UNICEF and WHO should include pediatric surgery in their activities including training and infrastructure development especially in developing countries to design the future for the children.

\section{References}

1. Black RE Morris SS, Bryce J. . Where and why are 10 million children dying every year? The Lancet 2003. 361 ( 9376): 2226-2234.

2. United Nations.2001. General Assembly, 56th session. Road map towards the implementation of the United Nations mi9llennium declaration: report of the Secretary-General (UN document no. A/56/326 ). New York: United Nations.

3. Lawn JE, Cousens S, Zupan J : Lancet Neonatal Survival Steering Team. 4 million neonatal deaths: when ? Where ? Why ? Lancet. 2005 Mar 2: 365 (9462): 891-900.

4. Mortality Country Fact Sheet 2006: World Health Statistics 2006.

5. State of the World Children, UNICEF 2009.

6. Ravitch MM, O'Neill JA and Rowe MI. 1998. Jejunoileal atresia. In Paediatric Surgery (O'Neill
JA, Rowe MI, Grosfeld JL, Fonkalsrud EW and Coran AG eds.), 5th edn., Vol. 2, pp. 1145-1158. Mosby-Year Book, Inc., St. Louis, Missouri.

7. Spitz L. The management of neonatal surgical emergencies. Progr Surg. 1987; 2: 18-33.

8. Spitz L. . Neonatal intestinal obstruction and intussusceptions in childhood. In Maingot's Abdominal Operations (Schwartz SI and Ellis H eds.), Appleton-Century-Crofts, New York. 1985; 2: pp.1054-1062.

9. Dickson JAS. Surgical emergencies in infants and older children. In Essentials of Paediatric Gastroenterology (Harries JT ed.), Longman Group Limited, London. 1977. pp. 63-104.

10. Gazzetta PC et al. Paediatric surgery. In Principles of Surgery (Schwartz SI, Shires GT and Spencer FC eds.), 5th edn., McGraw Hill Company, New York. 1988; pp. 1687-1727.

11. Neuenschwander S, Montagne JP, Boudou A, Bouron D, Mougenot JF, Gruner M and Faure C. Le "petit color gauch". Trois cas [The small left colon syndrome, 3 cases]. Nouv Presse Med. 1978; 5(35): 2311-2314.

12. Welch GH, Azmy AF and Ziervogel MA. The surgery of malrotation and midgut volvulus: A nineyear experience in neonates. Ann R Coll Surg Engl. 1983; 65(4): 244-247.

13. Reyes HM, Meller JL and Loeff D. . Neonatal intestinal obstruction. Clin Perinatol. 1989; 16(1): 85-96.

14. Paterson-Brown $\mathrm{S}$, Stalewski $\mathrm{H}$, and Brereton RJ. Neonatal small bowel atresia, stenosis and segmental dilatation. Br J Surg. 1991; 78 (1): 8386.

15. Murphree SH and Dunkley AS. Colon atresia and stenosis in Zimbabwe: Case reports and a review of the literature. Cent Afr J Med. 1992; 38(12): 463-465.

16. Kimura. $\mathrm{K}$ and Loening-Baucke $\mathrm{V}$. Bilious vomiting in the newborn: Rapid diagnosis of intestinal obstruction. Am Fam Physician. 2000; 61(9): 2791-2798.

17. The State of the World Children 2009, Maternal and newborn health: Where we stand, page 2-3.

18. Personal communications: Prof. Shafiqul Hoque, Prof. Tahmina Banu, Dr Ashraf-ul Huq 
19. Rahman SM, Talukder SA, Hanif A et al. Management of Omphalocele with Limited Facilities. J Dhaka Med coll. 2004; 13 (1): 58-62.

20. Bagolan P, Casaccia G, Crescenzi F, et al. Impact of a current treatment protocol on outcome of high-risk congenital diaphragmatic hernia. $\mathrm{J}$ Pediatr Surg 2004; 39: 313-8.

21. Hanif A, Hassan $K$, Hasina K, Rahman SM, Alam MM. 'Congenital Diaphragmatic Hernia (CDH) : Our experience of 21 cases in Dhaka Medical College Hospital, Dhaka'. Submitted for publication.

22. Nixon $\mathrm{HH}$. Congenital abnormalities of gut. $\mathrm{Br} \mathrm{J}$ Hosp Med. 1977; 11: 202-219.
23. Robertson J, Azmy AF and Young DG. Surgery in necrotizing enterocolities. Br J Surg. 1987; 4: 387-389.

24. Ameh EA and Nmadu PT. Intestinal atresia and stenosis: A retrospective analysis of presentation, morbidity and mortality in Zaria, Nigeria. West Afr J Med. 2000; 19(1): 39-42.

25. Landsman IS, Vustar M and Hays SR. Pediatric anesthesia. In Pediatric Surgery (O'Neill JA, Rowe MI, Grosfeld JL, Fonkalsrud EW and Coran AG eds.), 6th edn., Mosby-Year Book, Inc., St. Louis, Missouri. 2006; 1: pp. 221-256.

26. Hanif A, Hassan K, Hasina K et al. 'Neonatal Intestinal Obstruction: Six years experience in Dhaka Medical College Hospital, Bangladesh BJMS. 2009; 15(1): 2-45. 


\section{Up Coming Events}

\section{INTERNATIONAL SYMPOSIUM OF PEDIATRIC SURGERY}

Obergugl - Tyrol (Austria)

18th - 21st April 2010

\section{Topics}

- Chest wall deformities and complications

- Thoracic surgery

- Minimal invasive surgery

- Blunt abdominal trauma

- Urology

- Free papers

- Clinical research

- Interactive case presentations

- Video sessions

Deadline for abstracts: February 1, 2010

Organisation:

Univ.-Prof. Dr. Ernst Horcher

ernst.horcher@meduniwien.ac.at

Division of Pediatric Surgery - Medical University of

Vienna

General Hospital Vienna

Währinger Gürtel 18-20

1090 Vienna - Austria

Tel. 0043-1-40400-6836

Fax 0043-1-40400-6838
16TH WORLD CONGRESS OF PEDIATRIC AND ADOLESCENT GYNECOLOGY

22nd - 25th May, 2010

Montpellier, le Corum (France)

Congress information Congress information :

Congress President: Pr. Charles SULTAN c-

sultan@chu-montpellier.fr

Reduced fee for early registration:

December 14, 2009

Call For Abstracts

Free Communications

Online submission (www.figij2010.com)

Opening : September 15, 2009

Deadline for submission of abstracts : March 10, 2010

THE 46TH ANNUALCONGRESS OF THEJAPAN SOCIETY OF PERINATALAND NEONATALMEDICINE

July 11-13, 2010, Kobe, Japan

http://www.jspnm2010.jp/

3. 3rd World Congress of Pediatric Surgery

Oct 21-24,2010, New Delhi, India

\section{WOFAPS WORKSHOP - ADVANCES IN PEDIATRIC SURGERY}

November 23-25, 2010

Chittagong, Bangladesh

Contact : Prof. Tahmina Banu

E-mail:proftahmina@gmail.com

web site: www.pedsurgbd.org 